\title{
AMBER observations of the AGB star RS Capricorni: extended atmosphere and comparison with stellar models ${ }^{\star}$
}

\author{
I. Martí-Vidal ${ }^{1,2}$, J. M. Marcaide ${ }^{1}$, A. Quirrenbach ${ }^{3}$, K. Ohnaka ${ }^{2}$, J. C. Guirado ${ }^{1}$, and M. Wittkowski ${ }^{4}$ \\ ${ }^{1}$ Dpt. Astronomia i Astrofísica, Universitat de València, C/ Dr. Moliner 50, 46100 Burjassot, Spain \\ e-mail: imartiv@mpifr-bonn.mpg.de \\ 2 Max-Planck-Institut für Radioastronomie, Auf dem Hügel 69, 53121 Bonn, Germany \\ 3 Universität Heidelberg, Landessternwarte Königstuhl 12, 69117 Heidelberg, Germany \\ ${ }^{4}$ European Southern Observatory, Karl-Schwarzschild-Str. 2, 85748 Garching bei München, Germany
}

Received 10 November 2010 / Accepted 29 January 2011

\section{ABSTRACT}

\begin{abstract}
We report on $K$-band VLTI/AMBER observations at medium spectral resolution ( 1500) of RS Capricorni, an M6/M7III semi-regular AGB star. From the spectrally dispersed visibilities, we measure the star diameter as a function of observing wavelength from 2.13 to 2.47 microns. We derive a Rosseland angular diameter of $7.95 \pm 0.07$ mas, which corresponds to an effective temperature of $3160 \pm 160 \mathrm{~K}$. We detect size variations of around $10 \%$ in the $\mathrm{CO}$ band heads, which are indicative of strong opacity effects of CO in the stellar photosphere. We also detect a linear increase in the size as a function of wavelength, beginning at 2.29 microns. Models of the stellar atmosphere, based on the mass of the star estimated from stellar-evolution models, predict CO-size effects of about half the sizes observed, and cannot reproduce the linear size increase with wavelength, redward of 2.29 microns. We are able to model this linear size increase with the addition of an extended water-vapor envelope around the star. However, we are unable to fit the data in the CO bandheads. Either the mass of the star is overestimated by the stellar-evolution models and/or there is an additional extended $\mathrm{CO}$ envelope in the outer part of the atmosphere. In any case, neither the water-vapor envelope nor the $\mathrm{CO}$ envelope can be explained using the current models.
\end{abstract}

Key words. techniques: interferometric - stars: atmospheres - stars: late-type - stars: invididual: RS Cap

\section{Introduction}

The effective size of a star at a given wavelength depends on the opacity of the stellar atmosphere at that wavelength. Since we effectively measure the diameter of the $\tau=1$ surface of the star, the size is related to the extension of the atmospheric region where the absorption is produced. The diameter of that surface varies with opacity, hence frequency. The atmospheres of cool giants are so extended that these size variations are observable with an interferometer. Quirrenbach et al. (1993, 2001) studied the extended absorption regions of stellar atmospheres of cool giant stars in the TiO band at $712 \mathrm{~nm}$ with the MkIII interferometer. These authors measured the visibilities of a set of 47 stars using two filters, one centered in the $\mathrm{TiO}$ band, and the other in the continuum part of the spectrum close to the TiO band $(754 \mathrm{~nm})$. They found that the sizes in the $\mathrm{TiO}$ band are larger than those in the continuum, and that this effect is stronger for cooler stars. The sizes in the $\mathrm{TiO}$ absorption band are $\sim 10 \%$ larger than in the continuum for $R-I$ color indices of $\sim 1.6$ (spectral types M3M4) and as much as 30\% larger for $R-I$ color indices of $\sim 2.2$ (spectral types M6-M7).

Similar differences between the sizes observed in the continuum and in bands containing absorption band heads of other molecules (such as $\mathrm{H}_{2} \mathrm{O}$ or $\mathrm{CO}$ ) have been reported for AGB stars. Mennesson et al. (2002) and Perrin et al. (2004), for instance, reported very large size ratios $(50 \%$ or more) for some stars.

^ Based on observations made with ESO Telescopes at the Paranal Observatory under programme ID 383.D-0619.
Quirrenbach et al. (1993, 2001) were successful in qualitatively reproducing their data with the latest set of cool giant models from the general-purpose stellar atmosphere code PHOENIX. Spherical, hydrostatic, massively line-blanketed atmosphere models were constructed and used to predict the uniform-disk diameters in the $\mathrm{TiO}$ band and the continuum band as a function of model effective temperature, surface gravity, and mass (the stellar mass was used in the modeling, since it controls the deviation from plane-parallel atmospheres). For most of the observed oxygen-rich giants, the diameter ratios of the $\mathrm{TiO}$ band to the continuum band agreed with the models computed for masses $\sim 0.5 M_{\odot}$. Hence, model atmospheres with very low stellar masses could fit the large diameter ratios observed in many stars, although evolutionary models predict masses as high as $5 M_{\odot}$.

A possible explanation of this inconsistency would be the existence of a transition zone at the base of the stellar wind (the MOLsphere; e.g. Tsuji 2008, and references therein), which could provide sufficient opacity in the $\mathrm{TiO}$ bands (and other molecular bands) to make AGB stars appear much larger than predicted by the hydrostatic model atmospheres such as PHOENIX. According to this picture, one would expect similar size effects for the CO band heads. Therefore, we decided to use AMBER in the $K$ band to measure the effective sizes of a set of four cool giant stars through the CO band heads at $2.3 \mu \mathrm{m}$. The use of AMBER in medium-resolution mode $(\lambda / \Delta \lambda \sim 1500)$ provides considerably more information than could be obtained with the MkIII interferometer (Quirrenbach et al. 1993) and the IOTA interferometer (Mennesson et al. 2002; Perrin et al. 2004), 
Table 1. Details of our observations.

\begin{tabular}{|c|c|c|c|c|c|c|c|c|}
\hline & $\begin{array}{c}\text { UTC } \\
\text { (hh:mm) }\end{array}$ & $\begin{array}{c}\text { Seeing } \\
\left({ }^{\prime \prime}\right)\end{array}$ & Air mass & $\begin{array}{c}\text { Coh. time } \\
\text { (ms) }\end{array}$ & $\begin{array}{c}\text { E0-G0 } \\
L(\mathrm{~m})\end{array}$ & $\begin{array}{l}\text { G0-H0 } \\
L(\mathrm{~m})\end{array}$ & $\begin{array}{l}\text { E0-H0 } \\
L(\mathrm{~m})\end{array}$ & $\begin{array}{c}\text { PA } \\
\text { (deg) }\end{array}$ \\
\hline RS Cap & 07:08-08:06 & $0.61 / 0.94$ & $1.03 / 1.12$ & $1.6 / 2.4$ & $14.4 / 15.5$ & $28.7 / 31.0$ & $43.1 / 46.5$ & $119 / 113$ \\
\hline 18 Cap & $06: 47-06: 56 / 08: 16-08: 26$ & $0.57 / 1.19$ & $1.00 / 1.11$ & $1.5 / 2.7$ & $14.9 / 16.0$ & $29.7 / 31.9$ & $44.6 / 47.9$ & $110 / 123$ \\
\hline
\end{tabular}

Notes. For each star, we give the ranges of values obtained during all the exposures. $L(\mathrm{~m})$ is the projected baseline length (in meters) and PA is the position angle of the baseline (North through East). Coh. time is the coherence time estimated from the atmospheric conditions.

in which narrow-band filters were used. In this paper, we report on the results obtained from the analysis of the observations of the first star of our sample: RS Cap.

RS Cap (HD 200994) has a $K$-band magnitude of -0.2 (Cutri et al. 2003). It is a semi-regular variable (SRb) of spectral type M6/M7III and is located at $\alpha=21^{\mathrm{h}} 07^{\mathrm{m}} 15.4^{\mathrm{s}}, \delta=$ $-16^{\circ} 25^{\prime} 21.4^{\prime \prime}(\mathrm{J} 2000.0)$. It has a visual magnitude of 8.3 and a parallax $\pi=1.26 \pm 0.86$ mas (van Leeuwen 2007), which maps into a distance between 500 and 2500 pc. However, following Scalo (1976) or Winters et al. (2003), a bolometric absolute magnitude between -4 and -5 is derived for RS Cap (around -4.8 in the case of Winters et al.). These estimates, in addition to the spectrum fitted between 0.1 and $2 \mu \mathrm{m}$, translate into a distance of $\sim 300$ pc (Richichi et al. 1992).

A variability amplitude of $\Delta V \sim 0.5$ is seen in the Hipparcos data (Perryman \& ESA 1997), although a larger variability amplitude (in photometric magnitude) of $\Delta B \sim 2$ was reported in Kukarkin et al. (1969), which has a period of $~ 340$ days.

The remainder of this paper is structured as follows: in Sect. 2, we describe our AMBER observations and the strategy followed in the data calibration and reduction. In Sect. 3, we report on the results obtained: Sect. 3.1 is centered on our estimate of the effective temperature and Sect. 3.2 on the size effects observed at the CO band heads. In Sect. 4, we compare our results with synthetic data obtained from model stellar atmospheres. In Sect. 5, we summarize our conclusions.

\section{Observations and data reduction}

We observed RS Cap with the ESO Very Large Telescope Interferometer (VLTI) using the Astronomical Multi-BEam combineR, AMBER (see Petrov et al. 2007, for details on this instrument), in medium-resolution mode $(\lambda / \Delta \lambda \sim 1500)$. This instrument performs simultaneous observations of the interferometric fringes generated by three telescopes. Therefore, it measures closure phases, which are quantities independent of atmospheric or instrumental telescope-dependent contributions (e.g. Rogers et al. 1974). AMBER also measures the socalled differential phase on each baseline, which roughly represents the phase of spectral features with respect to that in the continuum $^{1}$. The observations were performed on 4 June 2009, from 06:30 UT to 08:30 UT, using the three VLTI Auxiliary Telescopes (AT) positioned on stations E0, G0, and H0. These stations are distributed roughly in the east-west direction. In Table 1, we give the projected baseline lengths and position angles for our observations, together with a summary of the atmospheric observing conditions. 18 Cap (M0III star located at $\alpha=20^{\mathrm{h}} 51^{\mathrm{m}} 49.3^{\mathrm{s}}, \delta=-26^{\circ} 55^{\prime} 08.9^{\prime \prime}, \mathrm{J} 2000.0$ ) was also

\footnotetext{
1 To be precise, two pieces of information are lost in the differential phase, compared to the original Fourier phase: the phase offset (or phase at the first spectral channel) and the phase gradient (phase as a linear function of wavenumber).
}

observed as a calibrator, with the same observing configuration used to obtain the RS Cap visibilities.

The high flux densities of RS Cap and 18 Cap in the $H$ band (magnitudes of 0.25 and 0.42 , respectively), allowed us to use the fringe tracker FINITO (see Gai et al. 2004), which employs part of the $H$-band light to correct, in real time, the delay shifts of the fringes due to atmospheric turbulences, thus increasing the coherence of the signal. A larger coherence allows the use of a longer detector integration time (DIT). Given the moderately good atmospheric conditions during our observations (see Table 1), we achieved a DIT of $200 \mathrm{~ms}$ with a negligible signal loss due to atmospheric jitter. This allowed us to simultaneously observe a large spectral range of the $K$-band (between 2.13 and $2.47 \mu \mathrm{m})$ using medium-resolution mode. This wavelength range contains the ${ }^{12} \mathrm{CO}(2-0),(3-1)$, and (4-2), as well as the ${ }^{13} \mathrm{CO}$ (2-0) band heads, together with the continuum blueward of the ${ }^{12} \mathrm{CO}(2-0)$ band head at $2.29 \mu \mathrm{m}$.

The data acquisition was carried out in the following order: (1) observation of an artificial signal for calibration of the instrumental dispersive effects; (2) one exposure of dark frames (an exposure consisted on 200 frames of $200 \mathrm{~ms}$ each); (3) five exposures of the target; and (4) one exposure of empty sky close to the target. The observations of dark, target, and sky exposures were iterated once for $18 \mathrm{Cap}$ (spanning $10 \mathrm{~min}$ ), three times for RS Cap (spanning $1 \mathrm{~h}$ ), and one more time for 18 Cap (spanning $10 \mathrm{~min}$ ). The dead time between iterations, when the sources were not observed, was dedicated to the setup of the instrument: preparation of the optical-delay lines, telescope pointing, etc.

The raw visibilities and differential closure phases for RS Cap and 18 Cap were obtained using the amdlib libraries (version 2.2) and the interface provided by the Jean-Marie Mariotti Center (JMMC). First, we manually aligned the spectrallydispersed photometry channels of the ATs to the interferometric channel, based on observations of the target and calibrator stars. We removed the bad pixels and took into account the flat, dark, and sky contributions. Afterwards, we calibrated the instrumental dispersive effects and fringe-fitted each frame of RS Cap and 18 Cap (based on the P2VM algorithm of Tatulli et al. 2007). The resulting visibilities of individual frames were selected and averaged for each exposure in several ways, to test the robustness of our results on different averaging and selection schemes. The finally chosen selection scheme was based on an atmospheric piston criterion (keeping only frames with a piston smaller than $8 \mu \mathrm{m}$, to select only well-detected and centered fringes in all three baselines) and keep 50\% of the remaining frames based on a signal-to-noise ratio (SNR) criterion.

After the frame selection, we obtained a single frameaveraged visibility spectrum for each exposure. The last step in the data reduction was to average the visibilities of all exposures of each star to increase the SNR. This step was performed outside amdlib, with an in-house developed python-based program that uses the PyFITS library (provided by the Space Telescope Science Institute, operated by AURA for NASA). The averaged closure phases and differential phases (i.e., those resulting from 
the averaged exposures) are zero within $3^{\circ}$, in absolute value, for both stars and for all the spectral channels. These small values of the closure phases indicate that the emission from both sources is symmetric, at least in the direction of the projected baselines of our observations and to the scale corresponding to our spatial resolution. This is unsuprising, given that we probe only the first lobe of the Fourier transform of the observed source structure, which is, therefore, only partially resolved.

The uncertainties in the amplitude and phase for each spectral channel were computed from their standard deviations during the averaging of the exposures. Fractional uncertainties of only $1-2 \%$ in the observed visibilities were obtained for all channels and baselines.

\subsection{Visibility amplitude and wavelength calibration}

A standard calibration procedure, to assign the corresponding wavelength to each spectral channel of the AMBER detector, is not currently available. This is a concern, especially for the mid-resolution and low-resolution modes, where it is difficult, or impossible, to use line profiles either from the observed objects or from Earth's atmosphere for the calibration. We performed the wavelength $(\lambda)$ calibration of our AMBER observations by comparing the positions of the minima of the observed $\mathrm{CO}$ band heads in RS Cap to their values derived from the models described in Sect. 4.1. The use of different stellar models did not affect our $\lambda$ calibration at a level higher than our spectral resolution.

In the amplitude calibration, the CHARM2 catalog of high angular-resolution stellar-diameter measurements (Richichi et al. 2005) indicates that the uniform-disk $K$-band diameter of our calibrator star, 18 Cap, is 5.02 mas. This uniform-disk diameter was used to calibrate the visibility amplitudes by comparing those of 18 Cap to the model predictions corresponding to a uniform disk of 5.02 mas diameter. This amplitude calibration was performed as described in Annex B of the AMBER Data Reduction Software User Manual (Revision 2.1) of the JMMC ${ }^{2}$.

\subsection{Photometry calibration}

In Fig. 1a, we show the normalized spectrum of RS Cap, obtained from the average of the photometry channels of the three ATs. We note that there is a clear discontinuity in the spectrum at $\sim 2.2 \mu \mathrm{m}$ (a similar discontinuity is also seen in the spectrum of 18 Cap). This discontinuity is caused by the transmission of the $K$-band fiber of the AMBER instrument. Therefore, it can be understood as an instrumental multiplicative effect, which biases the photometric and interferometric channels in the same way for wavelengths shorter than $\sim 2.2 \mu \mathrm{m}$. An additive contribution to these channels would have affected the contrast of the fringes relative to the photometry and, therefore, would have translated into a changing amplitude of the visibilities, which is not observed. Since any multiplicative bias in the data has no effect on the visibility amplitudes, the atmospheric opacity and any unmodeled gain effect in the detector do not affect the visibilities, but only the observed spectra.

If we wish to use the observed spectrum of RS Cap in our modeling, it is necessary to calibrate it with the atmospheric (and instrumental) spectral transmission profiles. To perform this calibration, we proceeded in the following way: 1) we obtained a template spectrum of an MOIII star, which is corrected for

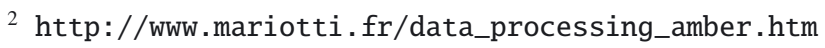

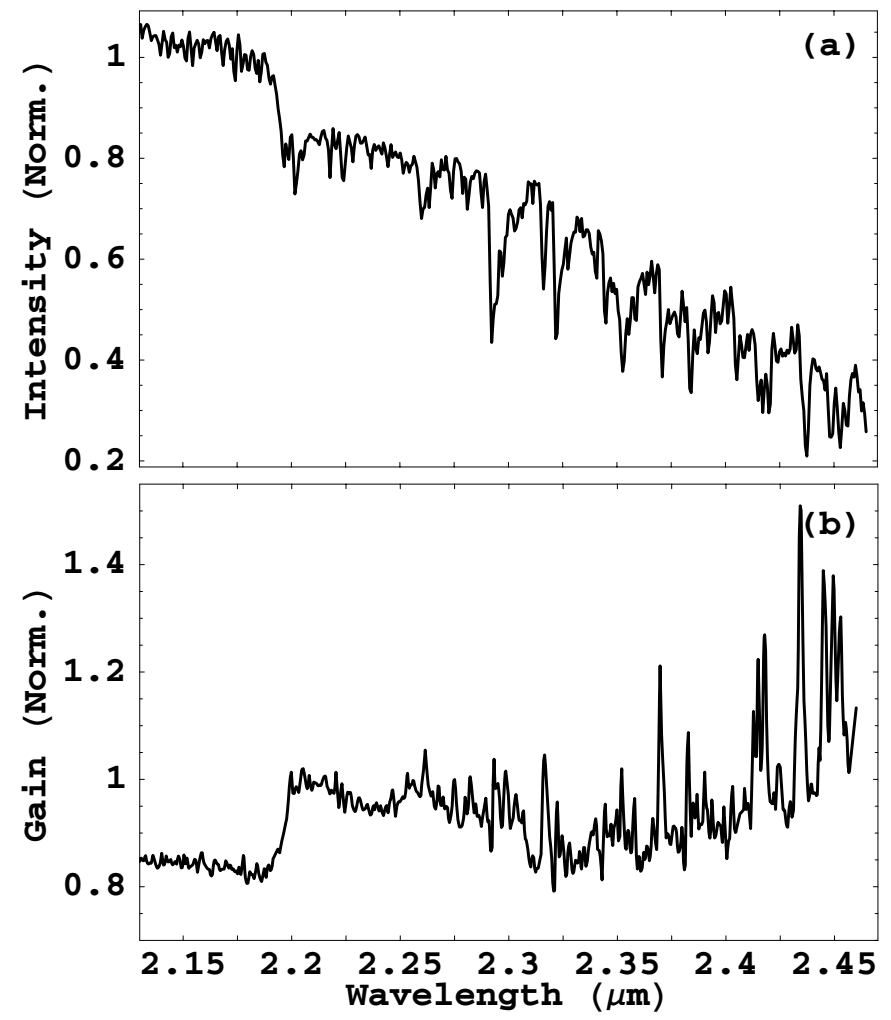

Fig. 1. a) Normalized spectrum of RS Cap, as observed with the ATs; b) inverse of the atmosphere+detector spectral transmission profile.

atmospheric effects (star BS4371, observed ${ }^{3}$ by Lançon et al. 2000); 2) we divided the template spectrum by the observed spectrum of 18 Cap, to obtain the inverse of the transmission profile of the atmosphere plus the detector (we call this quantity gain and we show it in Fig. 1b); and 3) we calibrated the spectrum of RS Cap, multiplying it by the gain. The normalized calibrated spectrum is shown in Fig. 2a. By applying this calibration strategy, we assume that the airmass is similar for target and calibrator, which is indeed the case in our observations (see Table 1).

We note that there are some atmospheric telluric lines that can be clearly seen in the transmission profile shown in Fig. 1b; those at 2.45 (three lines), 2.44, 2.42 (three lines blended), 2.37, and $2.32 \mu \mathrm{m}$. The effect of the optical-fiber transmission jump at $2.2 \mu \mathrm{m}$ is also seen in the gain, together with a slight increase as a function of wavelength redward of $\sim 2.35 \mu \mathrm{m}$ due to water vapor in the Earth's atmosphere. We also note that, although the CO bands might have slightly different depths in 18 Cap and BS4371, no clear features are seen in the regions of the $\mathrm{CO}$ bands for the gain shown in Fig. $1 b$.

\section{Results and discussion}

\subsection{Continuum angular diameter and effective temperature}

In Fig. 2, we show (a) the normalized spectrum of RS Cap, (b) the amplitude visibilities for the three baselines (higher visibility corresponds to shorter baseline), and (c) the resulting diameter estimates obtained from the fit of a uniformly-bright disk to the visibilities at each spectral channel.

The mean uniform-disk angular diameter fitted to the RS Cap visibilities in the continuum (i.e., blueward of $2.29 \mu \mathrm{m}$ ) is

3 This spectrum has a resolution of $\lambda / \Delta \lambda \sim 1100$, similar to that of our observations. 


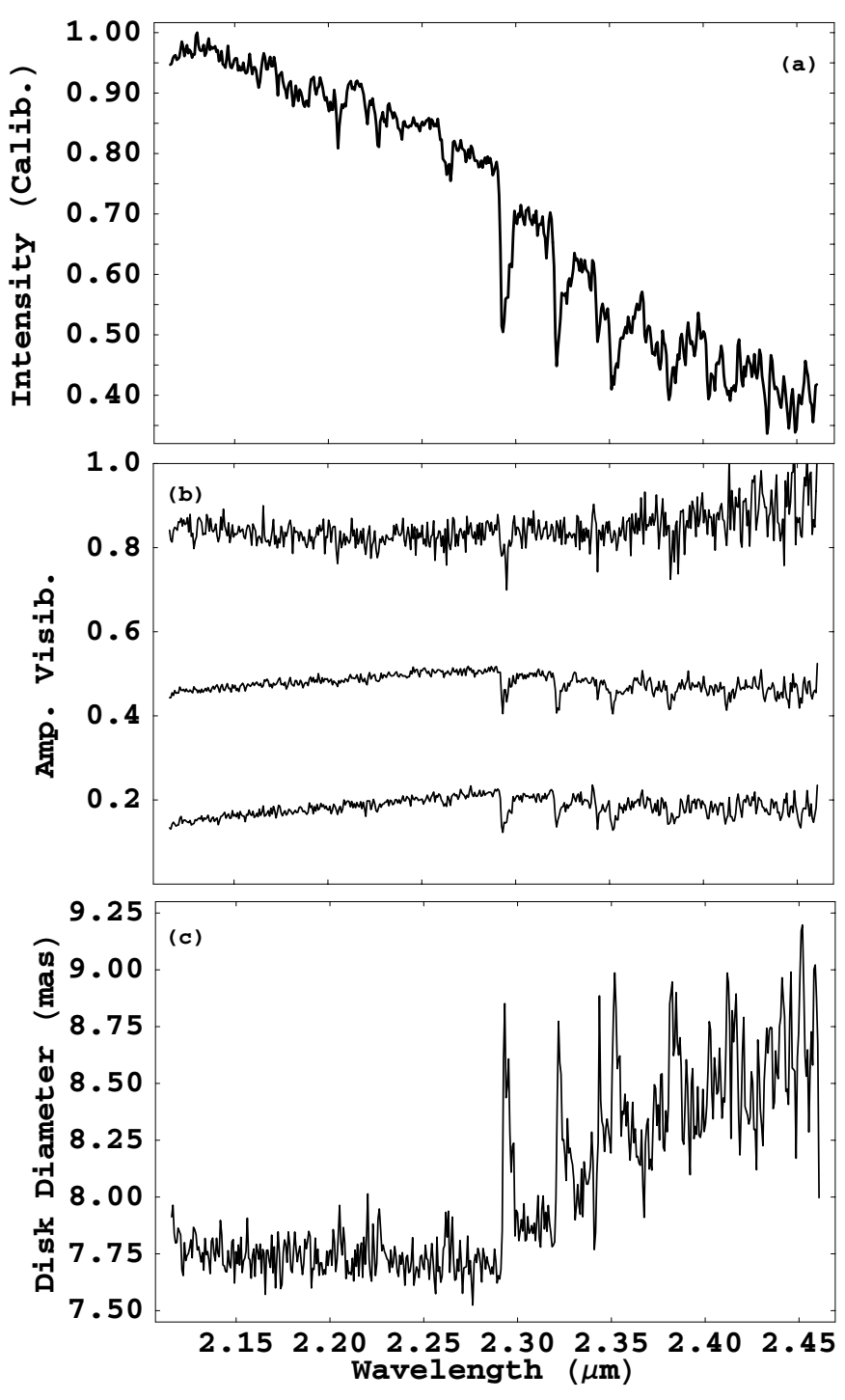

Fig. 2. a) Spectrum of RS Cap (normalized); b) calibrated visibility amplitudes; c) uniform-disc angular diameter at each AMBER spectral channel.

$\theta=7.75 \pm 0.07$ mas. To derive the effective temperature accurately, the size estimated with a limb-darkening model must be used, instead of that estimated from a uniform-disc model. We note that $T_{\text {eff }}$ is a function of the criterion adopted for the radius of the star. In spherical atmospheres, the actual $T_{\text {eff }}$ used to compare to the models should be computed using the diameter at which the Rosseland opacity is 1 (i.e., the Rosseland diameter, $\theta_{\text {Ross }}$; see, e.g., Sect. 3.4 of Wittkowski et al. 2004).

We estimated the Rosseland diameter of RS Cap by fitting the visibilities in the continuum part of our spectral coverage to the Hankel transform (i.e., Fourier transform of a circularlysymmetric source) of the monochromatic intensity profile of the MARCS atmospheric model described in Sect. 4.1. We show this intensity profile, normalized to a Rosseland radius $R_{\text {Ross }}=$ $\theta_{\text {Ross }} / 2=1$, in Fig. 3 . If this profile is given as $I(r)$ (where $I$ is the intensity normalized to 1 and $r$ is the radial coordinate normalized to the Rosseland radius, $R_{\text {Ross }}=1$ ), then the model used to fit the visibilities is

$V(q)=\int_{0}^{\infty} I\left(r / R_{\mathrm{Ross}}\right) J_{0}(K q r) r \mathrm{~d} r$

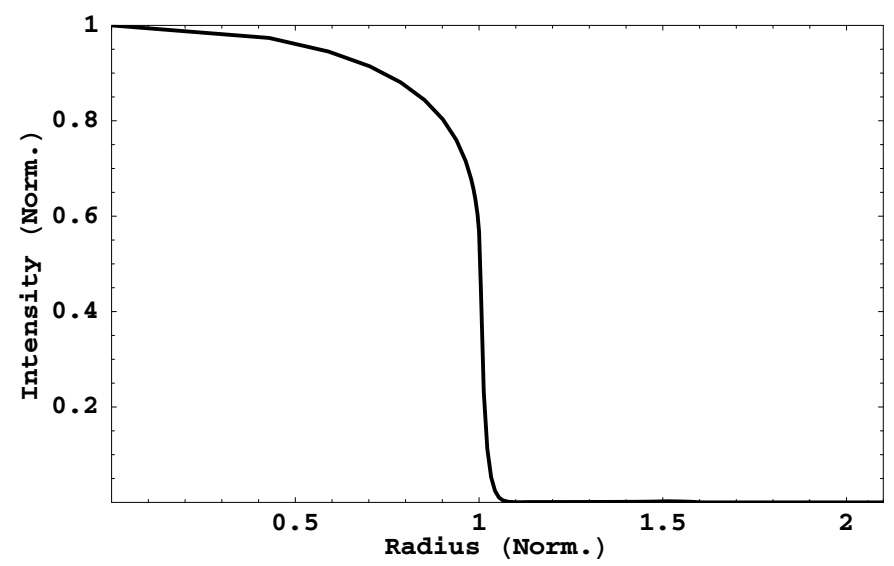

Fig. 3. Monochromatic intensity profile, normalized to $R_{\text {Ross }}=1$, corresponding to the MARCS model described in Sect. 4.1. This profile was computed in the continuum part of the spectral coverage of our observations.

where $J_{0}$ is the first-kind Bessel function of order $0, q$ is the baseline length, and $K$ is a constant to scale $r$ and $q$ to their corresponding units (e.g., milliarcseconds and megawavelengths, respectively). The use of this fitting model, instead of the Hankel transform of a uniform disc, allows us to take into account limb-darkening and, simultaneously, to estimate directly the Rosseland radius ( $R_{\text {Ross }}$ in Eq. (1)).

The best-fit Rosseland diameter of RS Cap is $\theta_{\text {Ross }}=7.95 \pm$ 0.07 mas. Our size estimate, together with the bolometric flux of $(2.1 \pm 0.2) \times 10^{-6} \mathrm{erg} \mathrm{cm}^{-2} \mathrm{~s}^{-1}$ reported in Richichi et al. (1992), translates into an effective temperature of $T_{\text {eff }}=3160 \pm 160 \mathrm{~K}$.

Lunar occultations of RS Cap, recorded with the TIRGO telescope at $2.2 \mu \mathrm{m}$, resulted in a uniform-disk diameter estimate of $7.75 \pm 0.67$ mas (Richichi et al. 1992). This diameter, together with the bolometric measurements performed by the same authors, implies an effective temperature of $\sim 3200 \mathrm{~K}$, higher than the temperature derived from the calibration of Bessel et al. (1998) ( 2900 K) and that derived from the calibration of Scalo (1976) ( 2600 K), but in agreement with the calibration given in Ridgway et al. (1980). A later re-analysis of the Lunar-occultation events (Richichi et al. 1999), to estimate the Rosseland angular diameter, resulted in an even higher effective temperature for this star $(3481 \pm 177 \mathrm{~K})$. Dyck et al. (1998) estimated a size of $7.0 \pm 0.8$ mas from a single-baseline interferometric observation in the $K$ band using the Infrared Optical Telescope Array (IOTA). This size also translates into a higher effective temperature for RS Cap (around $4000 \mathrm{~K}$ ). Our estimated diameter of RS Cap is compatible with that reported in Richichi et al. (1992) from lunar occultations and that reported in Dyck et al. (1998) from IOTA observations, although the precision in our estimate is an order of magnitude higher.

\subsubsection{A word of caution about the use of FINITO}

We note that the use of FINITO in our observations may bias the absolute amplitude calibration of the visibilities of each baseline, since the percentage of lost fringes during the DIT is not taken into account in the process of frame integration of each exposure. Any possible bias may be different for calibrator and target, and translate into a different absolute amplitude calibration for each star. As a result, our size estimate of RS Cap might be (slightly) biased even after performing the calibration described 


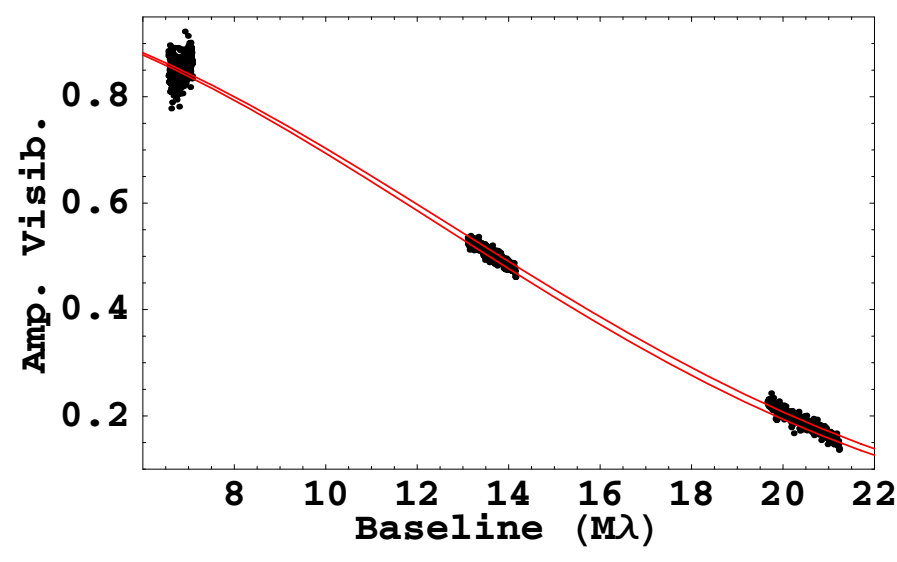

Fig. 4. Visibility amplitudes between 2.13 and $2.29 \mu \mathrm{m}$ as a function of baseline length. Black, observations; red, model predictions with a uniform-disk diameter of $7.75+0.07$ mas (lower line) and 7.75-0.07 mas (upper line).

in Sect. 2.1. However, the spectral resolution of our observations is much higher than that used in Richichi et al. (1992) and Dyck et al. (1998) (who, indeed, only measured the visibility at one projected baseline). The higher spectral resolution of AMBER allows us to precisely obtain the behavior of visibility amplitudes through relatively wide regions of the Fourier plane. This sampling of visibility amplitudes through the observing band encodes information about the source size, which is independent of the absolute calibration of the visibilities. In Fig. 4, we show the visibility amplitudes in the continuum (i.e. blueward of $2.29 \mu \mathrm{m}$ ) as a function of baseline length. In the figure, we also show the model predictions using a uniform-disk diameter of $7.75 \pm 0.07$ mas. It can be seen that a disk model with this diameter satisfactorily fits the visibilities of all baselines through the whole spectral coverage in the continuum. This result gives us confidence about the amplitude calibration of the RS Cap visibilities.

\section{2. $C O$ first overtone bands: $\lambda>2.3 \mu \mathrm{m}$}

In Fig. 2c, there are two clear aspects in the region of $\mathrm{CO}$ first overtone bands $(\lambda>2.3 \mu \mathrm{m})$ worth noticing. On the one hand, we see an increase of $\sim 10 \%$ in size for all the CO band heads, compared to the sizes in the continuum. On the other hand, there is a linear trend of increasing size with observing wavelength, beginning at $2.29 \mu \mathrm{m}$. Owing to this latter effect, the size in the continuum at $2.45 \mu \mathrm{m}$ is $\sim 12 \%$ larger than the size in the continuum for the wavelengths shorter than $2.29 \mu \mathrm{m}$.

Large size increases redward of $2.29 \mu \mathrm{m}$ have been observed in Mira stars (Woodruff et al. 2009), which are interpreted as being caused by water-vapor extended zones. However, in those cases, the size variations reported are 3-4 times larger than that observed in RS Cap. In Sect. 4.2, we discuss how our model of water-vapor envelope can explain the observed increasing size in RS Cap redward of $2.29 \mu \mathrm{m}$.

\section{Modeling}

\subsection{MARCS model atmosphere}

We compared the observed size effects in RS Cap with different model atmospheres computed with the MARCS code (e.g. Gustafsson et al. 2008). The MARCS code computes the hydrostatic atmospheric structure in radiative and convective equilibrium for spherical atmospheres with molecular and atomic lines taken into account using the opacity sampling method. Each model in spherical geometry (suitable for AGB stars) is specified by the effective temperature $\left(T_{\text {eff }}\right)$, surface gravity $(\log g)$, stellar mass $(M)$, microturbulent velocity, and chemical composition.

For $T_{\text {eff }}$, we adopted $3200 \mathrm{~K}$, the nearest possible value in MARCS models to our estimated $3160 \mathrm{~K}$. We also assume the "moderately $\mathrm{CN}$-cycled" chemical composition $(\mathrm{C} / \mathrm{N}=1.5$ and ${ }^{12} \mathrm{C} /{ }^{13} \mathrm{C}=20$ ), which is usually found in stars after the red giant branch, and $[\mathrm{Fe} / \mathrm{H}]=0.0$. The stellar mass can be estimated by comparing with theoretical stellar evolutionary tracks, once $T_{\text {eff }}$ and the luminosity are known. While the parallax of RS Cap is not precisely measured (the relative error is on the order of $70 \%$, so the error in the luminosity can be as large as an order of magnitude), Winters et al. (2003) estimated the luminosity of RS Cap to be $\log L / L_{\odot}=3.82$, by applying the period-luminosity relation for semi-regular variables given by Feast (1996) ${ }^{4}$. From Fig. 5 of Feast (1996), we estimated an uncertainty of \pm 0.25 in the bolometric absolute magnitude of RS Cap, which translates into an error of up to $20-25 \%$ in the luminosity, much smaller than that related to the Hipparcos parallax. The luminosity from Winters et al. (2003), together with the above $T_{\text {eff }}$, places RS Cap close to the evolutionary track of a $2 M_{\odot}$ star with $Z=0.02$, computed by F Herwig ${ }^{5}$. Therefore, we adopted $2 M_{\odot}$ for RS Cap.

We note that the luminosity estimated by Winters et al. (2003) for RS Cap is much higher than that corresponding to an RGB star with $2 M_{\odot}$ (which is only $\log L / L_{\odot} \sim 3$ ). Therefore, according to its luminosity, RS Cap would be clasified as an AGB star, as it is indeed assumed in the P-L relation by Feast (1996). The luminosity estimated in this way for RS Cap is, thus, selfconsistent in the framework of the P-L relation of semi-regular AGB stars.

From the luminosity and $T_{\text {eff }}$, we estimated a stellar radius of $\sim 260 R_{\odot}$, which, combined with our angular-size estimate, translates into a distance of about $310 \mathrm{pc}$. Hence, from the adopted stellar mass of $2 M_{\odot}$ and the estimated radius, we derive $\log g \sim-0.1$. We adopted $\log g=0.0$ for the selection of the MARCS models. For the microturbulent velocity, we used the MARCS models with 2 and $5 \mathrm{~km} \mathrm{~s}^{-1}$. However, the effects of this parameter on the visibility are very small.

We downloaded the models with the above parameters from the MARCS website ${ }^{6}$. Using the temperature and pressure stratifications of the model, we computed the monochromatic intensity profiles and the synthetic spectrum for the spectral range of our AMBER observations with a fine wavelength mesh of $1.4 \mathrm{~km} \mathrm{~s}^{-1}$, sampling several points over each line profile. The $\mathrm{CO}\left({ }^{12} \mathrm{C}^{16} \mathrm{O},{ }^{12} \mathrm{C}^{17} \mathrm{O},{ }^{12} \mathrm{C}^{18} \mathrm{O}\right.$, and $\left.{ }^{13} \mathrm{C}^{16} \mathrm{O}\right)$ and $\mathrm{H}_{2} \mathrm{O}$ lines were taken from the line lists of Goorvitch (1994) and Partridge \& Schwenke (1997), respectively. The monochromatic visibilities were computed from the Hankel transform (i.e., Fourier transform of a circularly-symmetric distribution) of the monochromatic intensity profiles (computed as described in Ohnaka et al. 2006) and then convolved to the spectral resolution of our AMBER measurements.

In Quirrenbach et al. (2001), limb-darkened photospheric models were used to generate equivalent disk sizes. These sizes

\footnotetext{
${ }^{4}$ Although the period-luminosity relation is a matter of debate, as Winters et al. (2003) mention.

5 http://astrowww.phys.uvic.ca/ fherwig

${ }^{6}$ http://marcs.astro.uu.se
} 
were then compared to the disk sizes fitted to the MkIII visibilities. In our case, this approach would have had the disadvantage of being sensitive to scaling factors between different baselines (i.e., to the absolute amplitude calibration) and would have depended on the use of an intermediate model (a uniform disk) to compare observations to models. To avoid those drawbacks, we chose a new approach to the modeling of the RS Cap visibilities, which minimizes the effect of possible calibration artifacts between baselines and, in addition, avoids the use of an intermediate fit to a disk. The synthetic visibilities obtained from the MARCS models were computed at the points of the Fourier plane corresponding to each exposure in our observations. We then averaged the synthetic visibilities over all the exposures, in the same way as we did with the real data. In this way, we directly compare the observed visibilities to the model predictions.

We show in Fig. 6 a comparison between observed visibilities (black lines) and those computed from the MARCS model using $T_{\text {eff }}=3200 \mathrm{~K}, M=2 M_{\odot}$, and $\log g=0$ (blue lines). The match between observations and model predictions in the continuum (i.e., for wavelengths shorter than $\sim 2.3 \mu \mathrm{m}$ ) is very good.

The reduced $\chi^{2}$ for this model is $\chi_{\text {red }}^{2}=18.2$. The relatively large depth of the features of the $\mathrm{CO}$ band heads cannot be reproduced by the model. The discrepancy remains even if we lower the mass to values as low as $M=1 M_{\odot}$ (models with higher masses predict more compact atmospheres and, therefore, smaller size effects in the CO band heads). Additionally, the observed visibility amplitudes at wavelengths longer than $\sim 2.29 \mu \mathrm{m}$ are systematically lower than the model predictions; this difference between model and observations is larger at longer wavelengths. In other words, the effective size of the star at wavelengths longer than $\sim 2.29 \mu \mathrm{m}$ is systematically larger than the effective size derived from the MARCS model.

\subsection{An additional water-vapor envelope}

An extra contribution must be added to the MARCS models to fit the visibilities in the observed spectral range. We can reproduce the lower visibility amplitudes (i.e., the larger effective sizes) beyond $2.29 \mu \mathrm{m}$ by adding a contribution to the stellar opacity due to water vapor around the star. The opacity corresponding to the wide absorption of water vapor centered at $\sim 2.7 \mu$ m may account for the larger angular sizes observed at the longer wavelengths.

We added a simple water-vapor model envelope to the MARCS pressure/density profiles, consisting of a narrow spherical shell with a radius of 2 and a width of 0.1 (both in units of the stellar radius), a column density of $10^{21} \mathrm{~cm}^{-2}$, and a temperature of $1500 \mathrm{~K}$. Using these parameters, we are able to fit the amplitude visibilities at $\lambda>2.29 \mu \mathrm{m}$, as we show in Fig. 6 (red lines). With this model, we obtain $\chi_{\text {red }}^{2}=8.1$. This large reduced $\chi^{2}$ is partially due to the discrepancy in the $\mathrm{CO}$ bands, which is discussed in Sect. 4.3. Our model follows the general trend of decreasing visibility amplitudes for wavelengths longer than $2.3 \mu \mathrm{m}$, although underestimates the visibility amplitudes at wavelengths longer than $2.4 \mu \mathrm{m}$. Changing the column density by a factor of ten results in an increase of $\chi_{\text {red }}^{2}$ by a factor 2-3; a slight modification of the water-vapor temperature $(\sim 15 \%)$ increases $\chi_{\text {red }}^{2}$ by a factor $\sim 2$; and changing the size of the shell by a factor of two increases the $\chi_{\text {red }}^{2}$ by a factor $\sim 2$.

Although the water-vapor envelope enhances the contrast of the $\mathrm{CO}$ features in the visibilities, thus improving the fit also in the $\mathrm{CO}$ band heads, the observed depths in the band heads are still larger than those of the model for some band heads $(\sim 50 \%$

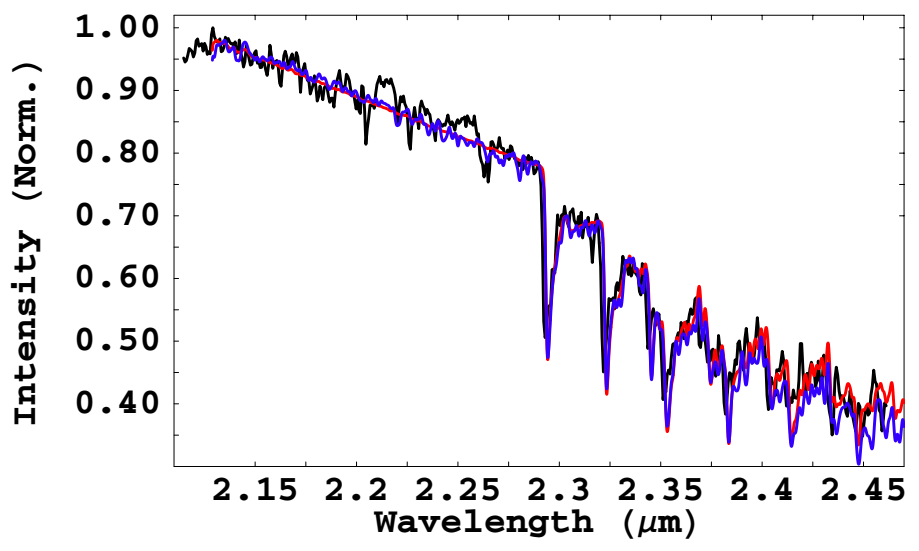

Fig. 5. Calibrated spectrum of RS Cap (black), synthetic MARCS spectrum (blue), and synthetic spectrum with the additional water-vapor envelope (red).

for the band head at $2.29 \mu \mathrm{m}$ ). Furthermore, increasing (decreasing) the stellar mass results in smaller (larger) depths of the visibility amplitudes in the $\mathrm{CO}$ band heads, but decreasing the mass to a value as low as $1 M_{\odot}$ does not translate into $\mathrm{CO}$ visibility amplitudes comparable to those of the observations, even after the addition of the water envelope (the $\chi_{\text {red }}^{2}$ using $1 M_{\odot}$ is only $7.2 \%$ lower than that using $\left.2 M_{\odot}\right)$.

In Fig. 5, we show the calibrated spectrum of RS Cap (black) and, superimposed, the synthetic spectra obtained from the MARCS model only (blue) and the MARCS model with an additional water-vapor envelope (red). Both model spectra are very similar, and only differ for the longest wavelengths, where the model with water envelope predicts a slightly lower flux. Since the fit of both models to the observed spectrum is of similar quality, it is difficult to extract any additional conclusion about the water-vapor envelope from the spectrum alone.

\subsubsection{Water-vapor envelopes in AGBs. The problem of RS Cap}

The presence of dense water vapor envelopes in semi-regular or irregular (i.e., non-Mira-type) AGB stars was first revealed by Tsuji et al. (1997), who unmasked the $2.7 \mu \mathrm{m}$ water vapor band originating in the dense molecular layers extending to $\sim 2$ stellar radii. On the basis of infrared interferometric observations, Mennesson et al. (2002) found an increase in the angular size of 30-60\% between the $K$ and $L^{\prime}$ band in five semi-regular AGB stars, which the authors interpreted as being due to the emission from the extended molecular layers. However, they used filters which are incapable of spectrally resolving the water vapor bands. Our AMBER measurements are the first study to spatially and spectrally resolve the water vapor emission from the dense molecular layers for a semi-regular AGB star.

We note that a similar increase in the angular diameter redward of $2.3 \mu \mathrm{m}$ is also found in Mira stars. Based on $K$-band interferometry using narrow-band filters with a spectral resolution of 20 , Perrin et al. (2004) showed that the angular diameters of Mira stars are larger at 2.0 and $2.4 \mu \mathrm{m}$ than at $2.2 \mu \mathrm{m}$. Wittkowski et al. (2008) observed the Mira star S Ori from 1.29 to $2.32 \mu \mathrm{m}$ using AMBER with a spectral resolution of 35 and found that the angular size increases redward of $2.2 \mu \mathrm{m}$. The wavelength dependence of the angular size of Mira stars from the near- to mid-infrared is primarily governed by the opacity of water vapor, 

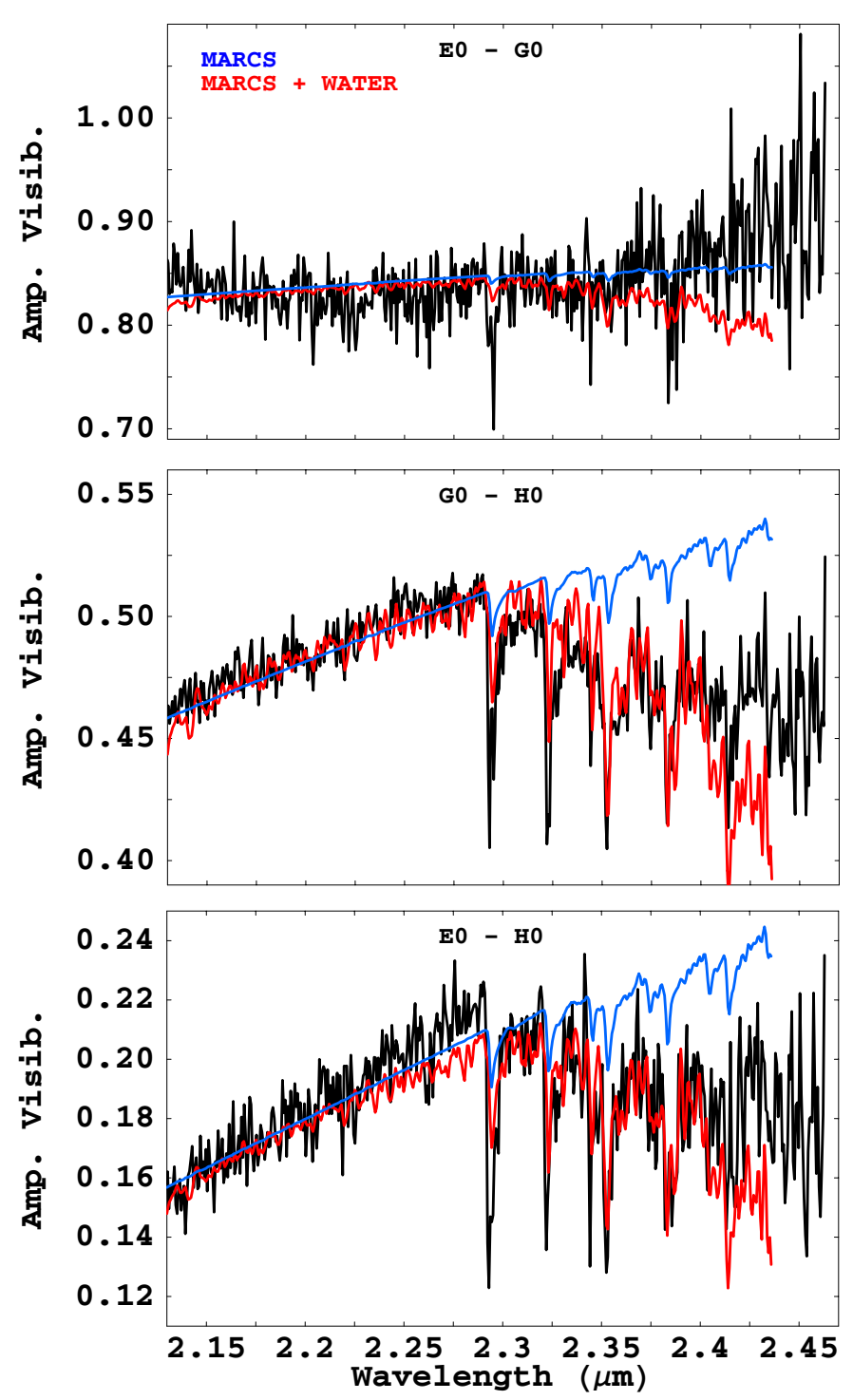

Fig. 6. Black: AMBER visibilities of RS Cap. Blue: model visibilities computed using a MARCS pressure-density profile (see text). Red: model visibilities computed using the MARCS pressure-density profile and an additional water-vapor envelope (see text).

which is abundant in the atmosphere of Mira stars (e.g., Ohnaka 2004; Wittkowski et al. 2008). The model by Tej et al. (2003) shows that a shell with an enhanced water vapor abundance forms behind a shock front propagating outward in the extended atmosphere of Mira stars. Comparison between infrared interferometric observations of Mira stars and dynamical models lends support to this picture (e.g., Ohnaka et al. 2006; Wittkowski et al. 2007).

The shock wave in Miras is generated by the periodic, largeamplitude pulsation. Therefore, the shock in non-Mira stars with much smaller variability amplitude is expected to be much weaker. However, despite the striking difference in the variability amplitude $(\Delta V=6-9$ and $0.5-2$ for Miras and RS Cap, respectively), the semi-regular AGB star RS Cap produces a warm water vapor envelope similar to that of Miras. The column density, temperature, and radius of the water vapor envelope derived for RS Cap are similar to those derived for Mira stars (Ohnaka 2004). Not only the properties of the water-vapor envelope, but also the mass-loss rate of RS Cap $\left(\sim 1 \times 10^{-6} M_{\odot}\right.$ $\mathrm{yr}^{-1}$ ) is comparable to that of Miras (e.g., $5 \times 10^{-7} M_{\odot} \mathrm{yr}^{-1}$ for $\mathrm{S}$ Ori) (estimates from Winters et al. 2003).

The dynamical models with small-amplitude pulsations, reported in Winters et al. (2003), produce outflows slower than $5 \mathrm{~km} \mathrm{~s}^{-1}$ with mass-loss rates lower than $3 \times 10^{-7} M_{\odot} \mathrm{yr}^{-1}$. However, the expansion velocity measured for RS Cap is $\sim 10 \mathrm{~km} \mathrm{~s}^{-1}$ (Winters et al. 2003). Therefore, it is not clear whether this kind of small-amplitude pulsation model applies to RS Cap. At the moment, there is no satisfactory explanation for the origin of the warm water envelope in RS Cap.

\subsection{An additional $C O$ envelope}

It can be seen in Fig. 6 that the observed visibility amplitudes at the $\mathrm{CO}$ band heads are much lower than the model predictions, either with or without a water-vapor envelope. This discrepancy is especially large for the shortest baseline (i.e. $E 0-G 0$ ) at the $2.29 \mu \mathrm{m}$ band head. The low visibility amplitudes at the CO band heads provide evidence of an extended envelope of $\mathrm{CO}$ around the star. For instance, if $\sim 20 \%$ of the emission in the CO band head at $2.29 \mu \mathrm{m}$ were to originate from a very extended envelope of, say, 20 mas radius or larger, it would map into a decrease in the visibility amplitude that would roughly match the observations in that part of the spectrum. Unfortunately, we do not have enough data to be able to characterize well this possible extra component of $\mathrm{CO}$ absorption.

\section{Conclusions}

We have observed the AGB star RS Cap with VLTI/AMBER in the $K$ band with medium-resolution mode (506 channels between 2.13 and $2.47 \mu \mathrm{m}$ ). We have estimated a Rosseland diameter of $7.95 \pm 0.07$ mas in the continuum, which translates into an effective temperature of $3160 \pm 160 \mathrm{~K}$. The apparent size of the star increases monotonically by $\sim 12 \%$ between 2.29 and $2.47 \mu \mathrm{m}$. We have detected lower than expected visibility amplitudes in all the $\mathrm{CO}$ band heads observed. These lower amplitudes translate into larger apparent sizes of the star in the $\mathrm{CO}$ band heads.

Using the pressure/density model profiles obtained with the MARCS code, we have been able to generate synthetic visibilities and compare them directly to our AMBER observations. The fit is rather good, although we are unable to reproduce the low visibility amplitudes in the $\mathrm{CO}$ band heads using a mass for RS Cap estimated from the theoretical models of stellar evolution. In this sense, our situation resembles that of Quirrenbach et al. (2001), who observed interferometrically a sample of cool-giant stars in the $\mathrm{TiO}$ absorption band at $712 \mathrm{~nm}$. The discrepancy between models and observations in the $\mathrm{CO}$ band heads might be resolved either by using a much lower mass for RS Cap (below $1 M_{\odot}$, thus in contradiction with the stellar-evolution models) or by additional unmodeled effects in the base of the stellar wind (a transition zone with prominent absorption in the $\mathrm{CO}$ bands).

To fit the observations at wavelengths longer than $2.29 \mu \mathrm{m}$, we have found that an ad hoc narrow spherical water-vapor envelope around the star, similar to the models used in Perrin et al. (2004), must be added. We have modeled this envelope with a temperature of $1500 \mathrm{~K}$, a size twice that of the star, a width of 0.1 times the stellar radius, and a column density of $10^{21} \mathrm{~cm}^{-2}$.

Finally, there is a hint of an extended $\mathrm{CO}$ envelope around the star, based on the low visibility amplitudes at the CO band heads in the shortest baseline. 
Acknowledgements. I.M.V. is a fellow of the Alexander von Humboldt Foundation in Germany. This research has made use of the SIMBAD database, operated at CDS, Strasbourg, France. Partial support from Spanish grants AYA-2006-14986-C02, AYA2009-13036-C02-02, and Prometeo 2009/104 is acknowledged.

\section{References}

Bessell, M. S., Castelli, F., \& Plez, B. 1998, A\&A, 333, 231

Claret, A. 2004, A\&A, 424, 919

Cutri, R. M., Skrutskie, M. F., van Dyk, S., et al. 2003, The IRSA 2MASS All-

Sky Point Source Catalog, NASA/IPAC Infrared Science Archive

Dyck, H. M., van Belle, G. T., \& Thompson, R. R. 1998, AJ, 116, 981

Gai, M., Menardi, S., Cesarec S., et al. 2004, The VLTI Fringe Sensors: FINITO and PRIMA FSU, SPIE Proc. 5491, Glasgow

Feast, M. W. 1996, MNRAS, 278, 11

Goorvitch, D. 1994, ApJS, 95, 535

Gustafsson B., Edvardsson B., Eriksson K., et al. 2008, A\&A, 486, 951

Kukarkin, B. V., Kholopov, P. N., Efremov, Yu. N., et al. 1969, General Catalog of Variable Stars, Academy of Sciences of the USSR, Moscow

Lançon, A., \& Wood, P. R. 2000, A\&AS, 146, 217

Manduca, A. 1979, A\&ASS, 36, 411

Mennesson, B., Perrin, G., Chagnon, G., et al. 2002, ApJ, 579, 446

Ohnaka, K. 2004, A\&A, 424, 1011

Ohnaka, K., Scholz, M., \& Wood, P. R. 2006, 446, 1119

Partridge, H., \& Schwenke, D. W. 1997, J. Chem. Phys., 106, 4618
Perrin, G., Ridgway, S. T., Mennesson, B., et al. 2004, A\&A, 426, 279

Perryman, M. A. C., \& ESA 1997, The Hipparcos and Tycho catalogues,

Noordwijk (The Netherlands: ESA Publications Division): ESA SP Ser., 1200 Petrov, R. G., Malbet, F., Weigelt, G., et al., A\&A, 464, 1

Quirrenbach, A., Mozurkewich, D., Armstrong, J. T., et al. 1993, ApJ, 406, 215

Quirrenbach, A., Mozurkewich, D., Armstrong, J. T., et al. 2001, BAAS, 33, 882

Richichi, A., Di Giacomo, A., Lisi, F., \& Calamai, G. 1992, A\&A, 265, 535

Richichi, A., Fabbroni, L., Ragland, S., \& Scholz, M. 1999, A\&A, 344, 511

Richichi, A., Percheron, I., \& Khristoforova, M. 2005, A\&A, 431, 773

Ridgway, S. T., Jacoby, G. H., Joyce, R. R., \& Wells, D. C. 1980, AJ, 85, 1496

Rogers A. E. E., Hinteregger H. F., Whitney A. R., et al. 1974, ApJ, 193, 293

Scalo, J. M. 1976, ApJ, 206, 474

Tatulli, E., Millour, F., Chelli, A., et al. 2007, A\&A, 464, 29

Tej, A., Lançon, A, \& Scholz, M. 2003, A\&A, 401, 347

Tsuji, T. 1988, A\&A, 197, 185

Tsuji, T. 2008, A\&A, 489, 1271

Tsuji, T., Ohnaka, K., Aoki, W., \& Yamamura, I. 1997, A\&A, 320, L1

van Leeuwen, F. 2007, Astrophysics and Space Science Library, 350

Weiner, J., Hale, D. D. S., \& Townes, C. H. 2003a, ApJ, 588, 1064

Weiner, J., Hale, D. D. S., \& Townes, C. H. 2003b, ApJ, 589, 976

Winters, J. M., Le Bertre, T., Jeong, K. S., Nyman, L.-Å., \& Epchtein, N. 2003, A\&A, 409, 715

Wittkowski, M., Aufdenberg, J. P., \& Kervella, P. 2004, A\&A, 413, 711

Wittkowski, M., Boboltz, D. A., Ohnaka, K., Driebe, T., \& Scholz, M. 2007, A\&A, 470, 191

Wittkowski, M., Boboltz, D. A., Driebe, T., et al. 2008, A\&A, 479, L21

Woodruff, H. C., Ireland, M. J., Tuthill, P. G., et al. 2009, 691, 1328 